\title{
Wi-Fi Based Indoor Next Location Prediction Using Mixed State-Weighted Markov-Chain Model
}

\author{
Jian Huang, Daniel Dahlmeier, Ziheng Lin, Boon-Khai Ang, Mun-Lie Seeto, and Hendy Shi
}

\begin{abstract}
Due to wide adoption of smart phones, Location Based Services (LBS) that leverage the user's location information have recently attracted an increasing amount of interest. As one of the most promising LBS technology, indoor Wi-Fi based positioning can provide relatively precise location information of Wi-Fi enabled mobile users at a low cost. In this paper, we propose a model called "Mixed State-Weighted Markov-chain Model" (MSWMM) to predict the next location of a user given his previous $\boldsymbol{n}$ locations, where the locations are derived from Wi-Fi based positioning. MSWMM is an improved version of the "Mixed Markov-chain Model" (MMM) and it takes into account the visited frequency of the same location and not just the transition probability between adjacent locations. In the experiment of comparing with MMM for $n=2$, MSWMM yields a significant $20.38 \%$ improvement of prediction accuracy over MMM.
\end{abstract}

Index Terms-Markov-chain model, next location prediction, Wi-Fi based positioning.

\section{INTRODUCTION}

Nowadays, Wi-Fi enabled connectivity has become one of the standard functions for smart phones and tablets with the trend of increasing number of indoor Wi-Fi enabled venues. For example, shopping malls provide free Wi-Fi connection to attract more shoppers. These free Wi-Fi services can bring more traffic to increase the visit volume of the shopping malls. Wi-Fi is also a "must-have" for retail stores [1]. Retailers want to be the special preference of their customers, in order to do that, they desire to have a direct relationship with their customers and remember their preferences. For example, once the customers come to their shops, these customers can be identified via Wi-Fi signal which is captured by their mobiles or tablets, a popup will be displayed in their mobile devices to inform them about new products or promotions and provide recommendations based on their historical purchase record.

In addition, in order to attract more customers into the shopping mall, these Wi-Fi services can also be used to help capturing indoor shoppers' location. Given a particular location, the smart phone can be represented by its Wireless LANs Address (MAC) uniquely and the received strength of the Wi-Fi signal indicator (RSSI) from different access points

Manuscript received April 9, 2014; revised July 8, 2014

Jian Huang, Boon-Khai Ang, Mun-Lie Seeto, and Hendy Shi are with the National University of Singapore, 25 Heng Mui Keng Terrace, 119615 Singapore (e-mail: A0092599@nus.edu.sg, A0092597@nus.edu.sg, A0092671@nus.edu.sg, A0092709@nus.edu.sg).

Daniel Dahlmeier is with SAP Asia Pte Ltd, CREATE Tower 1 Create Way, 138602 Singapore (e-mail: d.dahlmeier@sap.com).

Ziheng Lin is with Singapore Press Holdings, 1000 Toa Payoh North, 318994 Singapore (e-mail: linziheng@gmail.com). can be measured. By modeling the distribution of the RSSI in different locations, an Indoor Positioning System (IPS) as in [2], [3] can be built and trained with collected RSSI data to predict the device location from the signal strength. Although there are other options for indoor positioning, such as the indoor-messaging-system (IMES) in which positioning uses the indoor-GPS devices [4], however, because of the low-cost setup and the simple infrastructure needed, the Wi-Fi based IPS is more attractive to many retailers. Normally, the accuracy of these Wi-Fi Based IPS ranges from 3 to 10 meters [5]. If we keep recording the location of a MAC address for a period of time, these chronological location data can represent the movement or trajectory of a specified person.

In this work, we aim to develop a model to predict the pedestrian's next location based on his historical movement which can be represented by his trajectory. There are many potential ways in which users can benefit from this application of the next location prediction. By being aware of the movement of the shoppers in advance, the retailers can quickly target shoppers by both interest and location, turning window-shopping into actual sales [6]. In order to maximize the effect of coupon promotion, delivering the coupons in specified regions and at a particular time, namely "user oriented coupon dispersion", can be carried out. Leveraging the application of next location prediction, organizers can rearrange the manpower with more efficiency and at a lower cost.

This paper presents an improved model for the next location prediction and the remainder of this paper is organized as follows. First, we review relevant related works in Section II before describing Mixed Markov-chain Model (MMM) and its extension the Mixed State-Weighted Markov-chain Model (MSWMM) in Section III. Then we will report on the experimental comparison between MMM and MSWMM in Section IV before the concluding in Section V.

\section{RELATED WORKS}

In contrast to Outdoor Positioning System (OPS) such as global-positioning-system (GPS), Indoor Positioning System (IPS) has only become mature in recent years and started to emerge in commercial market. As a direct and promising application based on IPS, indoor next location prediction gradually attracts more attention but there is still not much related work on this topic whereas more research focused on the same application in the context of OPS have been published.

Gambs et al. [7], [8] proposed a Mobility Markov-chain Model (MMC) to incorporate the $\mathrm{n}$ previous visited locations and its extension coined as n-MMC to predict next possible 
visit location. In their work, the positioning data are coordinate values like ' $(x, y)=(198.3,85.1)$ ' which are collected from a smart phone or a GPS-equipped vehicle, these collected data will be processed by clustering algorithms such as the DJ-cluster to extract the Points Of Interests (POIs), so the trajectory of interest can be represented by a sequence of these POIs. Given enough trajectories of POIs for a user, a transition matrix which is named Mobility Markov-chain can be built by computing all the transition probabilities between each pair of POIs. The size of the transition matrix will be determined by the number of previous locations $\mathrm{n}$ and can be represented as (\# of POIs $)^{n+1}$. For example, if the number of POIs is 30 , when $n=1$, a $30 \times 30$ table is needed, and when $n=2$, a $30^{3}$ table will be needed instead, so it's not easy to perform larger $\mathrm{n}$ experiment due to the limited computational memory. In Gambs et al.'s evaluation of $n$-MMC, the accuracy of next location prediction is in the range of $70 \%$ to $95 \%$ at $n=2$. However, Ang et al. [9] conducted another evaluation of n-MMC in the indoor context and the prediction rate is only up to $49 \%$ when $n=2$. Two main realistic context differences lead to n-MMC cannot reach the similar prediction accuracy in indoor context as the one in outdoor context. Firstly, for Wi-Fi based IPS, it's easier that the Wi-Fi signal is reflected and absorbed when it encounters another medium with different electrical properties due to the limited room in which the signal propagates. So the instability of the Wi-Fi signal leads to the indoor positioning as in [9] is more difficult than the outdoor positioning as in [8]. The second also the most important reason is [8] build an individual model to predict where the specified one person to go, but [9] still use one single model to do the general prediction among different persons. The original design of n-MMC is used to model individual mobile trace but not for the general predictive purpose of crowds. So its ability of trace generalization will be limited in such indoor prediction context in which enough individual mobile traces may not be collected due to the behavior of users. For instance, generally, no one will go to the same shopping mall every day for several times, whereas most people need to go to work and come back home daily which can be represented via a fixed pattern. In one word, for the next location prediction of indoor context, a mixed model of higher trace generalization ability is needed.

A mixed Markov-chain Model (MMM) has been proposed for predicting pedestrians' next location by Asaharaet et al. [10]. Similar to Gaussian Mixture Models (GMM) [11], MMM is also a type of mixture component models in which each component is a compact representation on be behalf of a group of individuals. For MMM, these components are Markov-chains Models (MM) which are represented by transition matrixes. In order to generate and train these Markov-chains, given an amount of trajectories, Expectation Maximization (EM) algorithm can be performed to train the model iteratively on the purpose of increasing its overall likelihood to generate these trajectories. In the prediction stage, given a user's mobile trace of previous $\mathrm{n}$ locations, the goodness-of-fit between this user and each Markov-chain can be evaluated and finally next location can be calculated based on the fittest MM or top nth fittest MMs. Although, MMM gives a high prediction accuracy of $74.4 \%$ in a physical environment [10], its positioning data are discrete which are coming from the special indoor-GPS devices, namely indoor-messaging-system (IMES), whereas our data are continuous and coming from Wi-Fi Based IPS. Moreover, MMM does not take into account the temporal factor of the visited frequency in the same location which can also affect the next location prediction.

The main contribution in this work is that we extend the MMM to Mixed State-Weighted Markov-chain Model (MSWMM) in which the temporal factor of the visited frequency in the same location can also be considered.

\section{LOCATION PREDICTION MODEL}

\section{A. Common Notations}

Each location $l$ hereafter can be denoted by a series of natural number $\{1,2,3, \ldots, z\}$, and the $k$-th trajectory of pedestrian can be denoted by a vector $D_{k}=\left\{d_{k, 1}, d_{k, 2}, d_{k, 3}, \ldots\right.$, $\left.d_{k, i}\right\}$, where $d_{k, i}=l$, means the $k$-th pedestrian $i$-th position is $l$. Also given a trajectory denoted by $D_{k}$, the probability of such trajectory can be denoted by $P\left(D_{k}\right)$.

\section{B. State-Weighted Markov-Chain Model}

In MMM, each component in the mixture is a conventional Markov-chain Model (MM) which can be represent by a transition matrix, each element in the matrix $\omega_{\mu v}$ denotes the probability from state $\mu$ to state $v$, since what we consider here is a discrete transition, each state in effect represents one location, so hereafter $\omega_{\mu v}$ is also used to represent the probability from location $\mu$ to location $v$, where $\sum_{v} \omega_{\mu v}=1$. The overall transition probability is therefore written as follow:

$$
P\left(D_{k}\right)=P\left(\left\{d_{k, 1}, d_{k, 2}, d_{k, 3}, \ldots, d_{k, i}\right\}\right)=\prod_{i} \omega_{d_{k, i} d_{k, i+1}}
$$

And the $\omega_{\mu v}$ can be estimated by following formula:

$$
\omega_{\mu v}=\frac{\sum_{k, i} f\left(d_{k, i}==\mu\right) f\left(d_{k, i+1}==v\right)}{\sum_{k, i, \xi} f\left(d_{k, i}==\mu\right) f\left(d_{k, i+1}==\xi\right)}
$$

where $f(x)$ is a function which accepts a boolean expression as input and returns 1 when the expression is true, otherwise returns 0 .

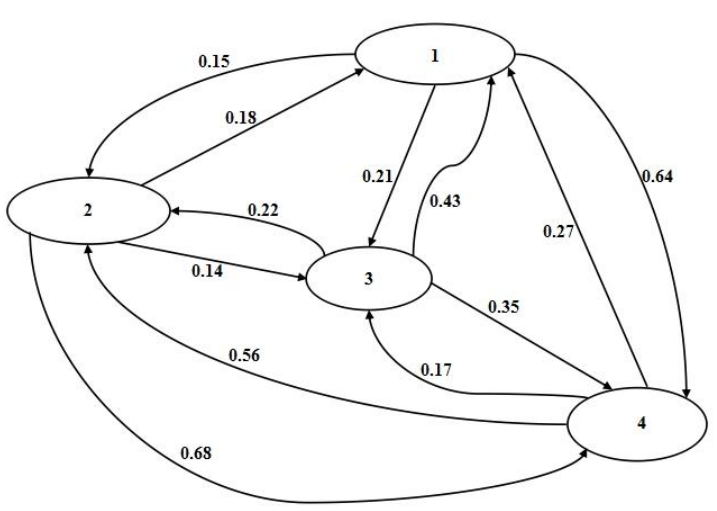

Fig. 1. Illustration of transition matrix in MM. 
The location transition matrix can also be represented by a directed Edge-weighted graph in which set of nodes connected by weighted edges as Fig. 1, in this description, each node represents a location and the weight of edge $e_{\mu v}$ denotes the probability from edge-head location $\mu$ to the edge-end location $v$. Since the number of the locations is fixed, $\sum_{v} e_{\mu v}=1$.

When considering the current scenario of the next location prediction, MMM does not take into account some other factors such the temporal changes of the movement probability. For instance, a customer visiting a specific shop frequently may imply that this customer also like to visit other shops of the same kind. Therefore based on given trajectory, following transition probability may be changed when the customers visits the same location at the second time onward. In order to capture these temporal changes during the movement, the node can be also assigned a weight based on the visit frequency of the location as Fig. 2 and this kind of the Markov-chain Model can be coined as State-weighted Markov Model (SWMM).

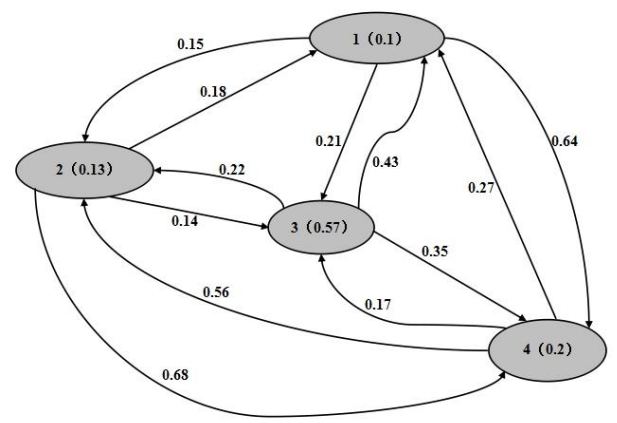

Fig. 2. Illustration of transition matrix in SWMM.

And the weight of node $\mu$ can be estimated as following formula:

$$
\rho_{\mu}=\frac{\sum_{k, i} f\left(d_{k, i}==\mu\right)}{\sum_{k, i, \xi} f\left(d_{k, i}==\xi\right)}
$$

Due to the states of SWMM having a weight, given a trajectory $D_{k}$, (1) can be revised as follow to estimate the overall transition probability of $D_{k}$ :

$$
P\left(D_{k}\right)=P\left(\left\{d_{k, 1}, d_{k, 2}, d_{k, 3}, \ldots d_{k, i}\right\}\right)=\prod_{i} \rho_{d_{k, i}} \omega_{d_{k, i} d_{k, i+1}}
$$

\section{Mixed State-Weighted Markov-Chain Model}

As a variant of MMM as in [10], Mixed State-Weighted Markov-chain Model (MSWMM) also contains an unobservable parameter for each pedestrian to determine which model generates the state transition and conform the same basic structure (see Fig. 3). Based on the assumption that the pedestrians in the same group have similar movement behavior, the trajectories of same group will share the same transition matrix. However, unlike MMM, the criteria to assign the category to trajectory do not just base on the transition matrix but also take into account how many times the previous known locations occurs in sense that the trajectories categorized into the same group will be more similar with each other and the generalization ability of
MSWMM is higher than the one of MMM. In one word, MSWMM use SWMM instead of MM as its component.

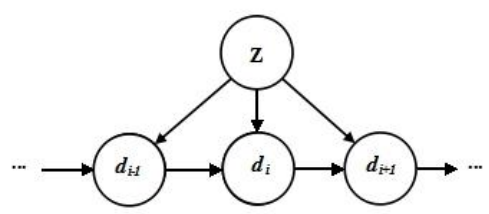

Fig. 3. Mixed State-weighted Markov-chain Model.

The probability-distribution formula used in MSWMM can be present as a latent vector $Z=\left\{z_{1}, z_{2}, z_{3}, \ldots, z_{k}\right\}$, and the $z_{k}=m$ means the $k$-th trajectory belongs to the $m$-th model. Since the latent parameter $Z$ and the observable parameters such as transition matrix need to be updated with each other simultaneously. Expectation-maximization (EM) [11] can be applied, and the EM algorithm for training MSWMM can be derived as follow:

$$
\begin{aligned}
& P\left(D_{k}, Z_{k}\right)=\sum_{z} P\left(D_{k} \mid Z_{k}\right) P\left(Z_{k}\right) \\
& =\sum_{m}^{M} \pi_{m} \prod \rho_{i} \rho_{m, d_{k, i}} \omega_{m, d_{k, i} d_{k, i+1}}
\end{aligned}
$$

where the $\pi_{m}$ is a mixing coefficient, and the $\omega_{m, d_{k, i}} d_{k, i+1}$ is the probability of the movement from location $d_{k, i}$ to location $d_{k, i+1}$ under the $m$-th model, the $\rho_{m, d_{k, i}}$ is the visit frequency ratio of location $\mathrm{d}_{k, i}$ under the $m$-th model. The following constraints are applied to the parameters:

$$
\sum_{m} \pi_{m}=1 \text { and } \sum_{\nu} \omega_{m, \mu \nu}=1 \text { and } \sum_{\mu} \rho_{m, \mu}=1
$$

The logarithmic likelihood $L$ then can be given as follow:

$$
L=\sum_{k} \log \sum_{m}^{M} \pi_{m} \prod_{i} \rho_{m, d_{k, i}} \omega_{m, d_{k, i} d_{k, i+1}}
$$

The objective of EM algorithm is to maximize $L$ under the constraint in (6) and finally it can be described as Fig. 4.

\section{Next Location Prediction by MSWMM}

Given a trajectory represented by $i-1$ previous visited locations, the most probable $i$-th location will obtained the maximum likelihood as following formula:

$$
\begin{aligned}
& \arg \max P\left(d_{k, i} \mid\left\{d_{k, i-1}\right\},\left\{\omega_{m, \mu v}\right\},\left\{\rho_{m, \mu}\right\},\left\{\pi_{m}\right\}\right) \\
& =\arg d_{k, i} \max \sum_{m}^{M} \gamma_{m}\left(d_{k, i-1}\right) \prod_{j \leq i} \rho_{m, d_{k, j}} \omega_{m, d_{k, j-1}} d_{k, j}
\end{aligned}
$$

\section{EXPERIMENTS}

\section{A. Settings}

In order to evaluate the prediction accuracy by MSWMM, experiments of MSWMM and MMM will be conducted and compared on the basis of the shoppers' tracking data collected in real environment. We installed a Wi-Fi based IPS in a department store in a shopping center. The IPS contains a set of Wi-Fi routers which covers the whole department store. When shoppers' Wi-Fi enabled smart phones and tablets come into the area covered by the IPS, its location together 
with time-stamp and MAC address will be saved into database in which each location is represented by a coordinate pair such as $(65.19,88.85)$. For our discrete transition model to handle these continuous numbers, we logically divide our studied area into 35 square zones in 5 rows $\times 7$ columns orientation as shown in Fig. 5. The actual size of each zone is $23.5 \mathrm{~m}^{2}$, and each zone will be assigned a number which is used to label the coordinates fall into it. Since the experimental IPS accuracy is around 10 meters, the zone of this size is tolerant enough to the fluctuation of the Wi-Fi signals. Before converting the continuous coordinate data into the discrete zone labels, we perform a data preprocessing upon the coordinates to filter out those adjacent coordinate pairs with movement speed exceeds the prefixed threshold $(1.2 \mathrm{~m} / \mathrm{s})$. For all the trajectories in hand, we compress successive location points sharing same label into a single occurrence and filter out those trajectories whose length is less than 9 to make sure that the trajectories to be used have enough duration. Finally, we get 12000 valid trajectories from which the data collected from February 2013 to June 2013.

Step 1. Initialization Initialize $\left\{\pi_{\mathrm{m}}\right\},\left\{\omega_{\mathrm{m}, \mu \nu}\right\}$, and $\left\{\rho_{\mathrm{m}, \mu}\right\}$ by random values.

Step 2. E-step Calculate responsibility $\gamma_{\mathrm{m}}\left(\mathrm{D}_{\mathrm{k}}\right)$ according to the following formula:

$$
\gamma_{m}\left(D_{k}\right)=\frac{\pi_{m} \prod_{i} \rho_{m, d_{k, i}} \omega_{m, d_{k, i} d_{k, i+1}}}{\sum_{m^{\prime}} \pi_{m^{\prime}} \prod_{i} \rho_{m^{\prime}, d_{k, i}} \omega_{m^{\prime}, d_{k, i} d_{k, i+1}}}
$$

Step 3. M-step Update the model parameter according to the following formula:

$$
\begin{aligned}
& \pi_{m}=\frac{1}{K} \sum_{k} \gamma_{m}\left(D_{k}\right) \\
& \omega_{m, \mu v}=\frac{\sum_{k, i} f\left(d_{k, i}==\mu\right) f\left(d_{k, i+1}==v\right) \gamma_{m}\left(D_{k}\right)}{\sum_{k, i, \xi} f\left(d_{k, i}==\mu\right) f\left(d_{k, i+1}==\xi\right) \gamma_{m}\left(D_{k}\right)} \\
& \rho_{m, \mu}=\frac{\sum_{k, i} f\left(d_{k, i}==\mu\right) \gamma_{m}\left(D_{k}\right)}{\sum_{k, i, \xi} f\left(d_{k, i}==\xi\right) \gamma_{m}\left(D_{k}\right)}
\end{aligned}
$$

Step 4. Convergence step Iterate E-step and M-step until convergence of likelihood occurs.

Fig. 4. EM algorithm for MSWMM.

\begin{tabular}{|c|c|c|c|c|c|c|}
\hline 1 & 2 & 3 & 4 & 5 & 6 & 7 \\
\hline 8 & 9 & 10 & 11 & 12 & 13 & 14 \\
\hline 15 & 16 & 17 & 18 & 19 & 20 & 21 \\
\hline 22 & 23 & 24 & 25 & 26 & 27 & 28 \\
\hline 29 & 30 & 31 & 32 & 33 & 34 & 35 \\
\hline
\end{tabular}

Fig. 5. Studied area.

Evaluation based on valid trajectories will adopt 5-fold cross validation. In the first step, the preprocessed tracking data set will be randomly partitioned into 5 equal size subsamples. Of the 5 subsamples, a single subsample is retained as the validation data for testing the model, and the remaining 4 subsamples are used as training data. The cross-validation process is then repeated 5 times, with each of the 5 subsamples used exactly once as the validation data. The 5 results from the folds can then be averaged to produce a single estimation. By means of the cross-validation, it can make the estimation result more reliable and guard it against the testing hypotheses suggested by the data (called "Type III errors") [12]. As the criteria to evaluate the model's predictive performance, the prediction rate can be given as follow:

$$
\begin{aligned}
& \text { Prediction Rate }(\%) \\
& =\frac{\text { Number of Correct Prediction Cases }}{\text { Total Number of Test Cases }} \times 100 \%
\end{aligned}
$$

\section{B. Cross-Validation Result}

The step-by-step prediction rates of the MMM and MSWMM are compared in Fig. 6. According to Fig. 6, MMM and MSWMM have the extremely close prediction rate by using $\mathrm{n}$ previous steps in which $\mathrm{n}$ is more than 2, while using 15 previous steps, MMM gives a prediction rate of maximum $72.17 \%$ while MSWMM gives $71.75 \%$. However, when using previous 2 steps, the MSWMM gives a prediction rate of $62.03 \%$, whereas MMM gives $51.53 \%$, MSWMM yields an increment of $(62.03 \%-51.53 \%) \times 100 / 51.53 \%=20.38 \%$ to the prediction rate over MMM and this improvement has been confirmed by 2 -tailed student t-test with $p<0.05$. Since using less previous steps, the prediction will be faster due to consume less computational resource, also less storage will be used to save the known locations, so if under the circumstances in which no more than 2 previous steps can be stored, MSWMM can be adopted instead of MMM with a higher prediction accuracy.

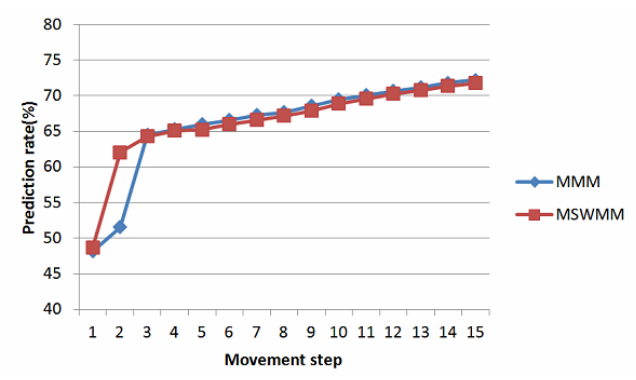

Fig. 6. Comparison of step-by-step prediction rates.

\section{CONCLUSIONS AND FUTURE WORK}

In this work, we propose a new prediction method based on the Mixed State-Weight Markov-chain Model (MSWMM), it significantly improve the prediction accuracy when using two previous steps which is compared with the one given by Mixed Markov-chain Model (MMM) in the Wi-Fi Based Indoor Positioning System (IPS) environment. Both models give similar performance with $n>2$. To improve the MSWMM further, more temporary changes of the mobility 
movement can be considered, for instance, the pattern of the movement in weekends may be different than the one in weekdays.

\section{REFERENCES}

[1] K. Jensen. (January 2014). Mall of America puts in Wi-Fi to drive in-venue experiences. [Online]. Available: http://www.mobilecommercedaily.com/mall-of-america-puts-in-wi-fito-drive-in-venue-experiences

[2] K. Tran, D. Phung, B. Adams, S. Venkatesh. "Indoor location prediction using multiple wireless received signal strengths," presented at the Seventh Australasian Data Mining Conference (AusDM 2008), Glenelg, Australia, 2008.

[3] D. L. Lee and Q. X. Chen, "A model-based wifi location method," in Proc. 2nd International Conference on Scalable Information Systems, no. 40, June 6-8, Suzhou, China, 2007.

[4] D. Manandhar, S. Kawaguchi, M. Uchida, M. Ishii, and H. Tomohiro. "IMES for mobile users. Social implementation and experiments based on existing cellular phones for seamless positioning," in Proc. the Int. Symposium on GPS/GNSS, Nov. 2008.

[5] D. Schneider. (November 2013). New indoor navigation technologies work where GPS can't. IEEE Spectrum. [Online]. Available: http://spectrum.ieee.org/telecom/wireless/new-indoor-navigation-tech nologies-work-where-gps-cant

[6] N. Clark and D. Dutch. (November 2013). Beyond retail: What's next for indoor location tracking with Apple's iBeacon. Venturebeat. [Online]. Available: http://venturebeat.com/2013/11/15/beyond-retail-whats-next-for-indo or-location-tracking-with-apples-ibeacon/

[7] S. Gambs, M.-O. Killijian, and M. Núòez del Prado Cortez, "Show me how you move and I will tell you who you are," Transactions on Data Privacy, vol. 4, pp.103-126, Catalonia, Spain, 2011.

[8] S. Gambs, M.-O. Killijian, and M. Núòez del Prado Cortez. "Next place prediction using mobility Markov chains," in Proc. the First Workshop on Measurement, Privacy, and Mobility, pp.1-6, April 10, 2012.

[9] B. -K. Ang, D. Dahlmeier, Z. Lin, J. Huang, M.-L. Seeto, and H. Shi. "Indoor next location prediction with Wi-Fi," in Proc. The Fourth International Conference on Digital Information Processing and Communications (ICDIPC), pp. 107-113, Kuala Lumpur, Malaysia, 2014.

[10] A. Asahara, K. Maruyama, A. Sato, and K. Seto, "Pedestrian-movement prediction based on mixed Markov-chain model," in Proc. 19th ACM SIGSPATIAL International Conference on Advances in Geographic Information Systems, pp. 25-33, 2011.

[11] C. M. Bishop, Pattern Recognition and Machine Learning, Singapore, Springer Science+Business Media, 2006, pp. 430-443.

[12] F. Mosteller, "A k-sample slippage test for an extreme population," Annals of Mathematical Statistics, vol. 19, no. 1, pp. $58-65,1948$.

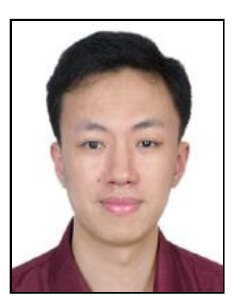

Jian Huang was born in China, 1985. He is pursuing the master of technology in knowledge engineering from National University of Singapore and expected to graduate in July 2014. He received his B.Eng from Jinan University (Guangzhou, China) in 2009.

He has been a software engineer and working in Singapore since 2010 . With over 4 years of experience in software design and development, he gets involved in different parts of the Software Development Life Cycle (SDLC) and multiple domains such as access control, intrusion detection and transportation. His current research interests include machine learning, distributed system.

Mr. Jian Huang is a graduate member of Singapore Computer Society.

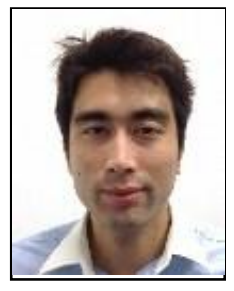

Daniel Dahlmeier was born on January 28, 1982 in Paderborn, Germany. He obtained his Ph.D. in computer science from the National University of Singapore in 2013 and prior to that his diploma in computer science from the Karlsruhe Institute of Technology, Germany.

$\mathrm{He}$ is currently a researcher at SAP Research \& Innovation in Singapore. His research interests are in the areas of machine learning and natural language

processing.

Dr. Dahlmeier is a member of the Association for Computational Linguistics. He received the Dean's graduate research excellence award and two research achievements awards from the NUS School of Computing.

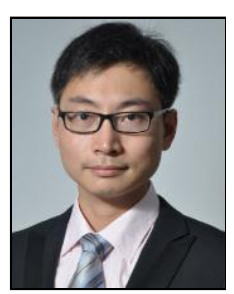

Ziheng Lin obtained his B.Comp degree and Ph.D. degree in computer science from School of Computing, National University of Singapore in year 2007 and 2012, respectively.

$\mathrm{He}$ is now a data scientist in Singapore Press Holdings, Singapore. Prior to that, he was a researcher in SAP Research \& Innovation, Singapore. He has published about 15 publications in top tier conferences and journals. His research interests include natural language processing, data mining, and information retrieval. Specifically, he has been working on discourse analysis, text coherence, text summarization, summarization system evaluation, opinion mining, and location mining.

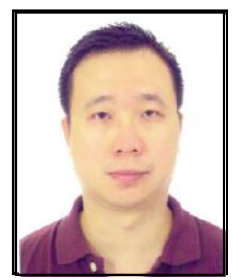

Boon-Khai Ang received the bachelor of science in microelectronics and physics from the Campbell University, USA, in 1998, and the master of engineering in telecommunication from the Multimedia University, Malaysia, in 2008.

$\mathrm{He}$ is currently a student at Institute of System Science in National University of Singapore. His research interests are in the areas of communication engineering and signal processing.

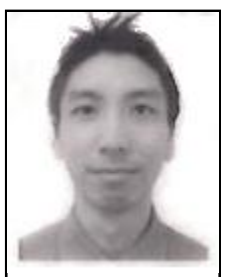

Seeto Mun Lie was born on February 6, 1982 in Singapore. He obtained his bachelor degree in computer engineering from the Nanyang Technological University in 2007 and master degree in knowledge engineering from Institute of System Science, National University of Singapore (NUS). He has done research in next step prediction for shopping mall during studies in NUS.

Technology Engineering.

$\mathrm{He}$ is currently a senior engineer at Singapore

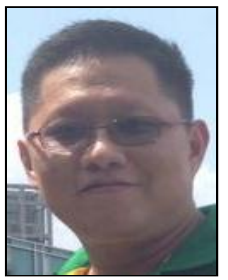

Hendy Shi Song Yuan was born in Indonesia and currently reside in Singapore on 14 September, 1971. He received his bachelor of science in physics instrumentation from University of Indonesia in November 1993. Currently he is pursuing master of technology in knowledge engineering from National University of Singapore and expected to graduate in July 2014.

He's been working in Singapore since graduation in 1993 till now. With over 20 years of experience in reliability, verification and validation, he understood customer need all the way from Design requirement all the way to customer experiences. He is involved in quality management system (QMS), supplier quality management, hardware quality, reliability and product regulatory compliance including safety, emc, environmental, wireless and country approval for medical devices, IT products. Currently he is working as the verification \& validation test lead in US Medical Device Company based in Singapore. 